\title{
SEASONAL VARIABILITY IN THE IONOSPHERE OF URANUS
}

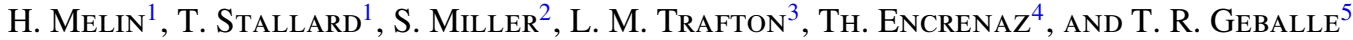 \\ ${ }^{1}$ Department of Physics \& Astronomy, University of Leicester, University Road, Leicester LE1 7RH, UK; h.melin@ion.le.ac.uk \\ 2 Atmospheric Physics Laboratory, Department of Physics \& Astronomy, University College London, Gower Street, London WC1E 6BT, UK \\ ${ }^{3}$ Department of Astronomy, The University of Texas, 1 University Station, C1400, Austin, TX 78712-0259, USA \\ ${ }^{4}$ LESIA, CNRS-UMR 8109, Observatoire de Paris, F-92195 Meudon, France \\ ${ }^{5}$ Gemini Observatory, 670 N. A'ohoku Street, Hilo, HI 96720, USA \\ Received 2010 November 16; accepted 2011 January 18; published 2011 February 18
}

\begin{abstract}
Infrared ground-based observations using IRTF, UKIRT, and Keck II of Uranus have been analyzed as to identify the long-term behavior of the $\mathrm{H}_{3}^{+}$ionosphere. Between 1992 and 2008 there are 11 individual observing runs, each recording emission from the $\mathrm{H}_{3}^{+} \mathrm{Q}$ branch emission around $4 \mu \mathrm{m}$ through the telluric $\mathrm{L}^{\prime}$ atmospheric window. The column-averaged rotational $\mathrm{H}_{3}^{+}$temperature ranges between $715 \mathrm{~K}$ in 1992 and $534 \mathrm{~K}$ in 2008, with the linear fit to all the run-averaged temperatures decreasing by $8 \mathrm{~K} \mathrm{year}^{-1}$. The temperature follows the fractional illumination curve of the planet, declining from solstice (1985) to equinox (2007). Variations in $\mathrm{H}_{3}^{+}$column density do not appear to be correlated to either solar cycle phase or season. The radiative cooling by $\mathrm{H}_{3}^{+}$is $\sim 10$ times larger than the ultraviolet solar energy being injected to the atmosphere. Despite the fact that the solar flux alone is incapable of heating the atmosphere to the observed temperatures, the geometry with respect to the Sun remains an important driver in determining the thermospheric temperature. Therefore, the energy source that heats the thermosphere must be linked to solar mechanisms. We suggest that this may be in the form of conductivity created by solar ionization of atmospheric neutrals and/or seasonally dependent magnetospherically driven current systems.
\end{abstract}

Key words: planets and satellites: atmospheres - planets and satellites: aurorae - planets and satellites: individual (Uranus) - planets and satellites: magnetic fields

\section{INTRODUCTION}

Although the four giant planets in our solar system are remarkably different from one another, Uranus presents itself as being particularly unusual. Much of our knowledge about this planet is derived from the solitary encounter of Voyager 2 in 1986, which revealed Uranus as an example of the extremes of physical parameters that a planet can assume: the rotational axis of Uranus is approximately aligned with the plane of the ecliptic, and its magnetic poles are positioned almost perpendicular to the axis of rotation. Thus, the magnetic field axis sweeps in large motions with the daily rotation of the planet $\left(P_{\mathrm{rot}}=17.24 \mathrm{hr}\right.$; Desch et al. 1986), which in combination with the relatively strong quadrupole and octupole field components (Connerney et al. 1987) creates what is likely to be a very complicated magnetic environment. It remains unclear exactly how the field interacts with the Interplanetary Magnetic Field (IMF) and what seasonal configurations, if any, are conducive for producing particle precipitation capable of ionizing the upper atmosphere.

Voyager 2 performed radio occultations of Uranus (Lindal et al. 1987) at both ingress and egress, observing the attenuation of the signal received at the Earth caused by atmospheric refraction. It observed sharp layers of electron conductivity up to $2000 \mathrm{~km}$ above the $1 \mathrm{bar}$ level. These sharp layers have been observed both on Jupiter (e.g., Fjeldbo et al. 1975, using Pioneer 10) and on Saturn (e.g., Kliore et al. 2009, using Cassini), but as of yet no satisfactory explanation for this phenomenon exists (see overview in Nagy et al. 2006). At higher altitudes, models indicate an ionosphere dominated by $\mathrm{H}^{+}$and $\mathrm{H}_{3}^{+}$(Capone et al. 1977; Chandler \& Waite 1986; Majeed et al. 2004), produced by either energetic particle precipitation or solar ultraviolet (UV) radiation.

The Voyager 2 Ultraviolet Spectrograph (UVS) solar and stellar occultations revealed an extended atmosphere with an exospheric temperature of $750 \mathrm{~K}$ (Broadfoot et al. 1986) - considerably hotter than the $400 \mathrm{~K}$ observed at Saturn (Sandel et al. 1982), but colder than the $1450 \mathrm{~K}$ observed at Jupiter (Broadfoot et al. 1981). This range of observed temperatures highlights how diverse a grouping the gas giants in our solar system are and that they must be subject to very different energy inputs.

Prior to the arrival of Voyager 2, Clarke (1982) observed a variation of a factor of two in the disk-integrated $\mathrm{H} \mathrm{Ly} \alpha$ emission using the International Ultraviolet Explorer (IUE). These observations were separated by $50.2 \mathrm{hr}$, equivalent to $30^{\circ}$ Uranian longitude, with each integration lasting $3.3 \mathrm{hr}$ during which time Uranus rotated by $\sim 70^{\circ}$. Since the IUE observations covered similar, overlapping, longitudes, they indicate the presence of a highly variable aurora, assuming a constant solar EUV contribution to the emission.

There has only been one spatially resolved observation of the aurora of Uranus (Herbert 2009), using a mosaic of Voyager 2 UVS observations, mapping emission from $\mathrm{H} \mathrm{Ly} \alpha$ and the Lyman and Werner $\mathrm{H}_{2}$ bands. While the signal-to-noise ratio $(\mathrm{S} / \mathrm{N})$ is only on the order of a few, the emission appears patchy and is generally centered on the magnetic poles, with the emission brightest about midnight magnetic local time. There have been subsequent attempts to observe the aurora in the UV using the Hubble Space Telescope (HST), but any auroral signatures remain undetected (Ballester et al. 1998). This casts some doubt on the IUE observations, since the sensitivity of $H S T$ ought to have been enough to match that of IUE.

Subsequent to the initial detection of $\mathrm{H}_{3}^{+}$emission from Jupiter (Drossart et al. 1989), the molecule has become an important instrument used to help understand both the properties of the upper atmosphere and how the ionosphere interacts with the magnetosphere at both Jupiter and Saturn (see overviews in Miller et al. 2000, 2006). In any large body of molecular 
hydrogen, such as the upper atmosphere of a gas giant, which is subjected to either radiation or particle energies large enough to ionize $\mathrm{H}_{2}, \mathrm{H}_{3}^{+}$is formed very efficiently via protonation of $\mathrm{H}_{2}$ by $\mathrm{H}_{2}^{+}$(forming a neutral $\mathrm{H}$ atom as well). Thus, in such an environment, $\mathrm{H}_{3}^{+}$becomes a dominant ion species and, since it is thermalized to the temperature of the neutral atmosphere, it becomes both a tracer of energy inputs and a probe for the physical conditions of the upper atmosphere.

$\mathrm{H}_{3}^{+}$emission was first detected from Uranus by Trafton et al. (1993). They derived a temperature of $757 \pm 25 \mathrm{~K}$ with a column-integrated density of $2.14 \times 10^{15} \mathrm{~m}^{-2}$ (Trafton et al. 1999). Lam et al. (1997) analyzed $3.9 \mu \mathrm{m}$ images of Uranus and concluded that auroral processes were responsible for not more than $20 \%$ of the total $\mathrm{H}_{3}^{+}$emission. The images had low $\mathrm{S} / \mathrm{N}$, but indicated a variable emission across the disk of the planet, consistent with auroral emission rotating in and out of view.

The most comprehensive study of $\mathrm{H}_{3}^{+}$emissions from Uranus to date is Trafton et al. (1999), presenting infrared spectra obtained during early to mid-1990s. They observed a decrease in the global $\mathrm{H}_{3}^{+}$emission of $30 \mathrm{GW}$ per year between 1992 and 1995 caused predominantly by a decrease in $\mathrm{H}_{3}^{+}$temperature. Trafton et al. (1999) speculated that this decrease was driven by the declining phase of the solar cycle, injecting less solar radiation into the upper atmosphere. In addition, they noted that the $\mathrm{H}_{3}^{+}$emission generally peaked on the center of the disk of Uranus, in contrast to $\mathrm{H}_{2}$ emission, which displayed an edge enhancement consistent with a thick emitting shell. This suggests that different mechanisms are responsible for exciting ionospheric emission from that producing the neutral emission.

Encrenaz et al. (2000) observed infrared emission from Uranus at $3.3 \mu \mathrm{m}$ using the Infrared Space Observatory (ISO), deriving an $\mathrm{H}_{3}^{+}$rotational temperature of $600 \pm 200 \mathrm{~K}$ and a column density of $(0.1-40) \times 10^{16} \mathrm{~m}^{-2}$, the upper range of which is much larger than the values derived by Trafton et al. (1999).

Ground-based observations have been successful in separating the signatures of the solar EUV and particle precipitation mechanisms producing $\mathrm{H}_{3}^{+}$emission for Jupiter (Satoh \& Connerney 1999) and, to a lesser extent, for Saturn (Stallard et al. 1999). This is because the magnetic fields of those two planets are closely aligned with their rotational axis, creating distinct, relatively localized, circumpolar ovals of auroral emission. As of yet for Uranus, however, it has not been possible to separate $\mathrm{H}_{3}^{+}$emission produced by solar radiation from that due to particle precipitation.

Here, we present a study that combines a large number of medium and high resolution infrared spectra of Uranus, covering the period between 1992 and 2008, during which the activity of the Sun declined to solar minimum, increased to solar maximum, and then declined once more. Monitoring the temperature and density of the $\mathrm{H}_{3}^{+}$ionosphere for periods exceeding the length of a solar cycle allows the possibility of gaining an insight into how it responds to changes in solar cycle phase as well as to seasonal changes in the geometry of Uranus with respect to the Sun. First, however, we briefly review factors that may effect the observed $\mathrm{H}_{3}^{+}$emission and the physical parameters we derive from it.

\section{DATA AND ANALYSIS}

Over the past two decades, observers at University College London, University of Texas at Austin, University of Leicester,

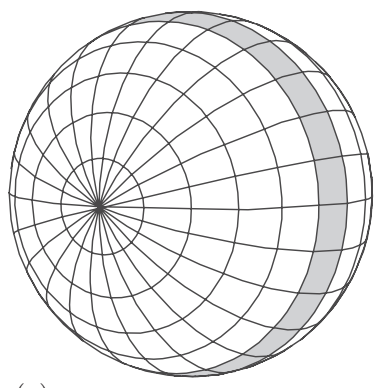

(a)

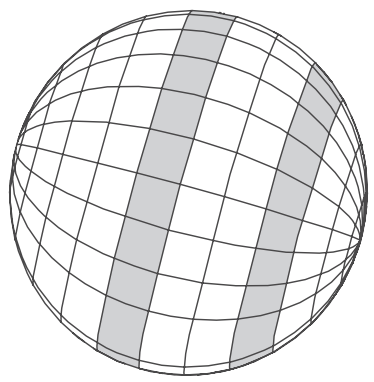

(b)
Figure 1. Geometry of Uranus in (a) 1992 and (b) 2008 as viewed from the Earth, with the IAU south pole (or northern ULS pole) to the left at both epochs. The shaded areas show the approximate latitude of the magnetic poles, which are separated by $\sim 160^{\circ}$ longitude (Herbert 2009).

Observatoire de Paris, and the Gemini Observatory have acquired a large set of infrared observations of $\mathrm{H}_{3}^{+}$emission from Uranus using ground-based telescopes. A sub-set of these observations is re-analyzed here. Our selection is based on two stringent criteria. First, for a particular observing run, a co-added spectrum must have a high enough $\mathrm{S} / \mathrm{N}$ to produce a fitted $\mathrm{H}_{3}^{+}$ temperature with an error $\left(\Delta T_{\text {err }}\right) \leqslant 50 \mathrm{~K}$ in order for any longterm temporal variability to be statistically significant. Second, the spectral range of the spectrum must contain at least some of the discrete $\mathrm{H}_{3}^{+} \mathrm{Q}$ branch emission lines in the 3.95-4.05 $\mu \mathrm{m}$ spectral region (see Figures 2-4), ensuring that each data set is analyzed in a consistent manner and that dependable spectral fits are obtained for each data set.

Trafton et al. (1999) saw an underpopulation in the $\mathrm{H}_{3}^{+}\left(v_{2}=\right.$ $2) /\left(v_{1}=1\right)$ derived temperature on Uranus, suggesting that there may be departures from local thermodynamic equilibrium (LTE) that affect the relative populations of the vibrational states. However, there are currently no atmospheric models capable of detailing the non-LTE effects on the $\mathrm{H}_{3}^{+}$in the atmosphere of Uranus, and no study of how rotational sublevels within a vibrational level might depart from LTE. The Q branch emission around $4 \mu \mathrm{m}$ has been shown to be the wavelength region least sensitive to small departures from LTE in the thermosphere of Jupiter (Melin et al. 2005), the only planet for which such a study has been undertaken. In order to minimize the possible non-LTE effects in a consistent manner in this study, therefore, only the Q-branch region in the Uranus $\mathrm{L}^{\prime}$ spectra is fitted. It is assumed, then, for the purposes of this analysis, that this spectral region behaves as if in quasi-LTE (q-LTE), as defined by Miller et al. (1990).

The geometry of Uranus as seen from the Earth in both 1992 and 2008 is shown in Figure 1, with the shaded areas indicating the latitude of the magnetic poles. Southern hemisphere summer solstice occurred in 1985 March, less than a year before the flyby of Voyager 2. Equinox occurred in 2007 December, such that winter solstice will occur in 2030 May. Due to the configuration of the magnetic field, the northern magnetic pole only became visible in the latter half of the 1990s.

The rotation period of Uranus was determined by Voyager 2 radio observations to be $17.24 \pm 0.01 \mathrm{hr}$ (Desch et al. 1986). More recently, Herbert (2009) determined it to be $17.21_{-0.04}^{+0.00} \mathrm{hr}$ by performing a $\chi^{2}$ fit of the Voyager UVS auroral observations to a rotation period, where $0.04 \mathrm{hr}$ is the step-size of the period distribution. This gives a relatively large range in the uncertainty of the Uranian rotation period of between $0.02 \mathrm{hr}$ and $0.08 \mathrm{hr}$, which means that for each telluric year, the uncertainty in subobserver longitude increases by between $212^{\circ}$ and $848^{\circ}$, such 
Table 1

The Observing Runs Available for the Long-term Analysis of the $\mathrm{H}_{3}^{+}$Emission from Uranus

\begin{tabular}{|c|c|c|c|c|c|}
\hline Run Number & UT Date & $\begin{array}{l}\text { Slit } \\
\left({ }^{\prime \prime}\right)\end{array}$ & $\begin{array}{c}\text { Resolution } \\
(\lambda / \Delta \lambda)\end{array}$ & Instrument & Previously Published \\
\hline 1 & 1992 Apr 1-2 & 3.1 & 1,300 & CGS4 (UKIRT) & Trafton et al. (1993) \\
\hline 3 & 1994 Jul 20/23 & 1.5 & 1,200 & CGS4 (UKIRT) & Trafton et al. (1999) \\
\hline 4 & 1995 Jun $11-14$ & 1.2 & 1,400 & CGS4 (UKIRT) & Trafton et al. (1999) \\
\hline 5 & 1999 Sep $14-18$ & 0.7 & 5,570 & CGS4 (UKIRT) & $\ldots$ \\
\hline 7 & 2001 Jun $16 / 18-19$ & 0.61 & 1,200 & CGS4 (UKIRT) & $\ldots$ \\
\hline 8 & 2001 Sep 2 & 0.8 & 2,500 & SpeX (IRTF) & Encrenaz et al. (2003) \\
\hline 9 & 2002 Jul 18-22 & 0.5 & 2,500 & SpeX (IRTF) & $\ldots$ \\
\hline 10 & 2006 Sep 05 & 0.28 & 25,000 & NIRSPEC (Keck II) & $\ldots$ \\
\hline 11 & 2008 Oct $19-21$ & 0.5 & 2,500 & SpeX (IRTF) & $\ldots$ \\
\hline
\end{tabular}

Table 2

Results of the $\mathrm{H}_{3}^{+}$Fitting Routine Applied to Uranus $L^{\prime}$ Spectra Listed in Table 1

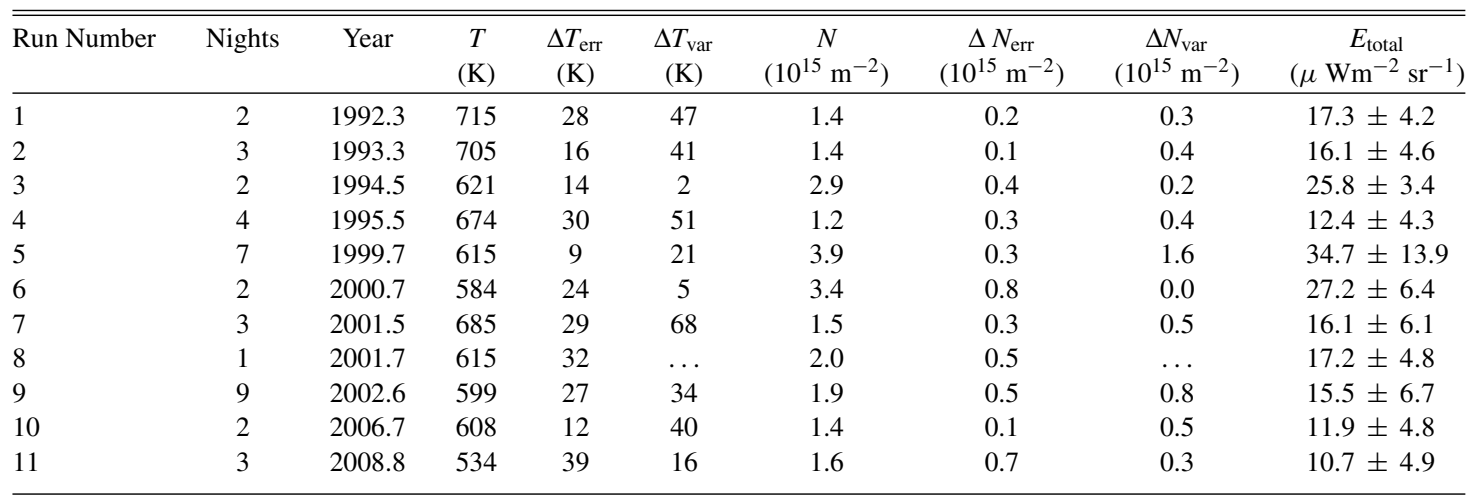

that for any observation subsequent to 1990, the sub-Earth longitude at the time of observation is an unknown quantity. Since the magnetic axis is not aligned with the rotational axis we cannot know in advance if an auroral region is in view for any given observation. If there were a prominent auroral component to the $\mathrm{H}_{3}^{+}$emission, the emission might be expected to display diurnal variability, with a period of either one (Figure 1(a)) or half of a rotation period (Figure 1(b)), depending on seasonal geometry. We will investigate diurnal variability in a later study.

For each of the observing runs listed in Tables 1 and 2, the $\mathrm{L}^{\prime}$ spectra of Uranus was dark-current subtracted, flat-fielded, flux calibrated and co-added to produce just one spectrum. To minimize flux calibration errors, standard stars were observed with wide $\left(\sim 3^{\prime \prime}\right)$ slits as well as the narrow slit used for obtaining the $\mathrm{H}_{3}^{+}$spectrum, ensuring that $\geqslant 95 \%$ of starlight was collected even when seeing conditions were $\sim 1^{\prime \prime}$. It is not possible to correct fully for changes in observing conditions in between flux standard observations. We estimate that these effects cause an uncertainty of $\sim \pm 10 \%$ in our absolute intensities. The resulting spectrum was fitted with a model $\mathrm{H}_{3}^{+}$spectrum (the fitting procedure is discussed below), giving a line-of-sight averaged temperature and column integrated density of $\mathrm{H}_{3}^{+}$. Using these two parameters the energy emitted over all wavelengths, $E\left(\mathrm{H}_{3}^{+}\right)$, can be calculated (Miller et al. 2010).

The observations in Table 1 are all obtained with different spectrograph configurations, some with the slit aligned along the central meridian (north-south) and some with the slit aligned parallel with the equator (east-west). Uranus has an angular diameter of $\sim 3^{\prime \prime} .7$. The slit widths used in our observations range from as little as 0 .'28 to 3 .' 1 , nearly matching the planet's diameter. Individual spectral exposures were all of the order of $60 \mathrm{~s}$. Thus, the instantaneous spatial coverage of each observation varied considerably. However, in order to integrate to get an $\mathrm{S} / \mathrm{N}$ greater than the $\Delta T_{\text {err }} \leqslant 50 \mathrm{~K}$ required to fit a spectrum with sufficient confidence, the total integration time is on the order of $4-5 \mathrm{hr}$, equivalent to half a night of observing for all CGS4 and SpeX observations (see below for discussion of NIRSPEC data). During such a period Uranus rotates through $83^{\circ}-105^{\circ}$ of longitude, and with several nights of observations, most longitudes will be sampled. Consequently, given consecutive nights of observation for each of the runs, each data set provides a measure of the longitude-averaged emission during that period, regardless of slit orientation and slit width. Trafton et al. (1999) observed an $\mathrm{H}_{3}^{+}$intensity profile across the disk of Uranus that peaked at its center, indicating no significant edge enhancements, which would be most affected by seasonal geometry.

There are two data sets, runs 8 and 10 , for which there is only one night of observation that can be fitted, which provide an only partial picture of the $\mathrm{H}_{3}^{+}$ionosphere. For the purposes of this paper, however, we assume that the column density and temperature derived for these observations are sufficiently representative for the globally averaged conditions for that epoch for our comparisons to be valid.

Six out of the 11 data sets have been published previously (see Table 1). Here, each of those sets of observations are re-analyzed with an improved $q$-LTE $\mathrm{H}_{3}^{+}$fitting routine and restricting the fit to the Q-branch at around $3.9 \mu \mathrm{m}$, to ensure consistency in 


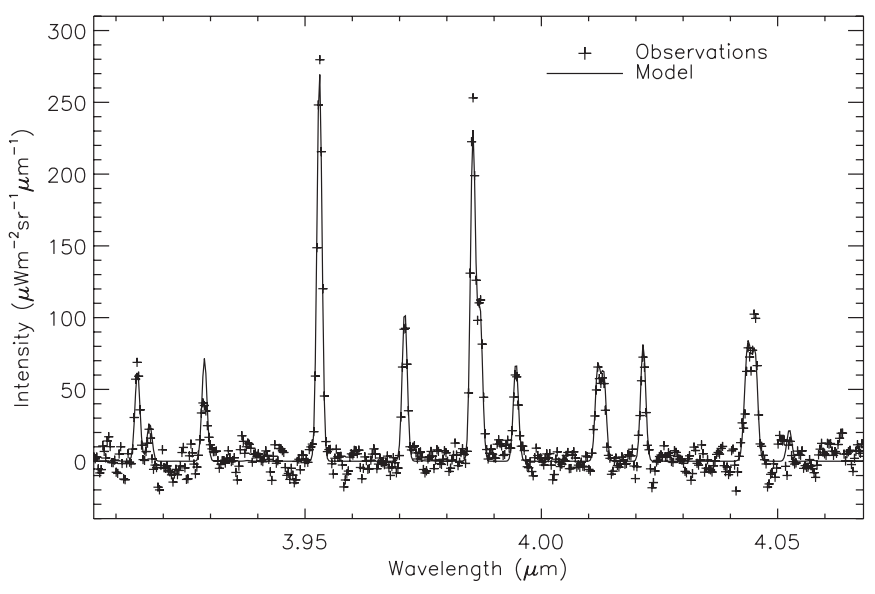

Figure 2. Co-added UKIRT CGS4 spectrum of Uranus from the 11th of June 1999 run (crosses). It shows the $\mathrm{H}_{3}^{+} \mathrm{Q}$ region around $4 \mu \mathrm{m}$ together with a $q$-LTE $\mathrm{H}_{3}^{+}$model fit (solid) of $T=605 \pm 8 \mathrm{~K}$ and $N=(3.1 \pm 0.2) \times 10^{15} \mathrm{~m}^{-2}$.

the analysis process. The following sections briefly outline the instruments used and the $\mathrm{H}_{3}^{+}$spectral fitting procedure.

\subsection{UKIRT CGS4}

Six of the 11 observing runs listed in Table 1 were obtained using United Kingdom InfraRed Telescope (UKIRT), a $3.8 \mathrm{~m}$ telescope operated by the Joint Astronomy Center (JAC). The Cooled Grating Spectrometer 4 instrument (CGS4; Mountain et al. 1990) has undergone two configurations since the first observation considered here. For the first set in Table 1, CGS4 had a $58 \times 62$ detector array installed and was used with a 150 lines $\mathrm{mm}^{-1}$ grating. For run 2, the same detector array was used but with a 50 lines $\mathrm{mm}^{-1}$ grating. For subsequent CGS4 observations, the spectrograph was equipped with a $256 \times$ 256 pixels detector array and a 40 lines $\mathrm{mm}^{-1}$ grating.

Note that a single grating can produce a range of resolving powers $(\lambda / \Delta \lambda$, listed in Table 1$)$, since it is a function of both central wavelength and the diffraction order that is used. A spectrum of Uranus taken with CGS4 in 1999 can be seen in Figure 2, clearly showing discrete $\mathrm{H}_{3}^{+} \mathrm{Q}$ branch line emission.

Trafton et al. (1993) reported the discovery of $\mathrm{H}_{3}^{+}$emission from Uranus using CGS4 spectra obtained on the 1st of April 1992 (run 1 in Table 1). This study includes data from the 2nd of April 1992, which is not included in Trafton et al. (1993).

\subsection{NASA IRTF SpeX}

There are four data sets in this study obtained with SpeX, a medium resolution $0.8-5.4 \mu \mathrm{m}$ spectrograph with a $1024 \times$ 1024 pixels detector array (Rayner et al. 2003). It is mounted on the $3 \mathrm{~m}$ NASA InfraRed Telescope Facility (IRTF). The SpeX long cross-dispersed (LXD) 1.9 mode produces a spectral image that contains several spectral orders, covering 1.9 to $4.2 \mu \mathrm{m}$ at a resolution of $R=2500$. A spectrum of Uranus taken with SpeX in 2008 can be seen in Figure 3.

\subsection{Keck II NIRSPEC}

Run 10 listed in Table 1 is the only data set with sufficient $\mathrm{S} / \mathrm{N}$ to enable a temporal resolution of less than half a night of observing. NIRSPEC (McLean et al. 1998) is mounted on the $10 \mathrm{~m}$ Keck II telescope and has a $1024 \times 1024$ pixels detector which in combination with the KL grating $(2.134-4.228 \mu \mathrm{m})$ and the $0^{\prime} .288 \times 24^{\prime \prime}$ high resolution slit gives pixel-scale

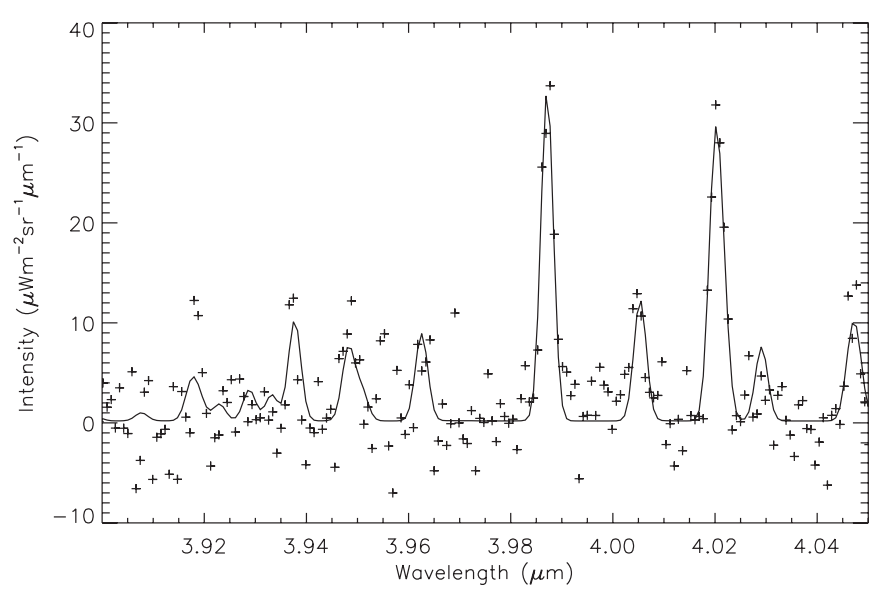

Figure 3. Co-added IRTF SpeX spectra of Uranus from 20th October 2008 (crosses). The solid line shows the $q$-LTE $\mathrm{H}_{3}^{+}$model fit to the observed spectrum with $T=518 \pm 35 \mathrm{~K}$ and $N=(1.9 \pm 0.8) \times 10^{15} \mathrm{~m}^{-2}$. This data set has the largest $\mathrm{S} / \mathrm{N}$ of the sets analyzed here while still satisfying $\Delta T<50$.

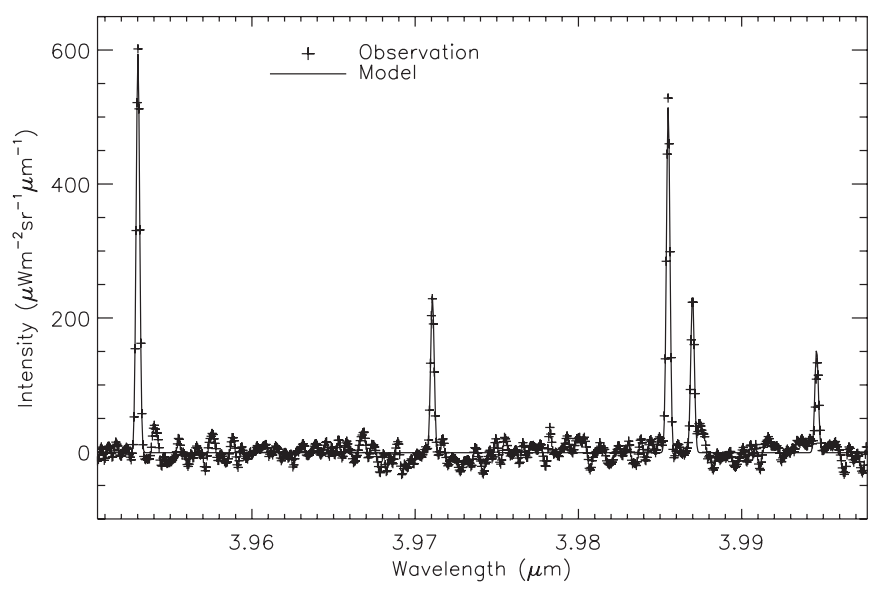

Figure 4. Single Keck II NIRSPEC spectrum of Uranus from the 5th of September 2006 (crosses), fitted with a $q$-LTE spectrum with $T=625 \pm 12$ and $N=(1.2 \pm 0.1) \times 10^{15} \mathrm{~m}^{-2}$ (solid).

of $0.144 \mathrm{pixel}^{-1}$ and a resolution of $R=25,000$. These observations of Uranus span only $3 \mathrm{hr}$, covering some $60^{\circ}$ longitude. However, although having incomplete longitude coverage, this data set adds a valuable constraint for the $\mathrm{H}_{3}^{+}$ temperature and column density in the period between 2002 and 2008 , close to equinox in late 2007. An individual spectrum (of $240 \mathrm{~s}$ ) of Uranus taken with NIRSPEC can be seen in Figure 4.

This rich data set will be explored in more detail in future studies.

\section{4. $H_{3}^{+}$Model Fitting Procedure}

The $\mathrm{C}++q$-LTE $\mathrm{H}_{3}^{+}$-spectral-fitting procedure, detailed in Melin (2006), was developed in an effort to remove the limitations of the existing routines, by including the option to fit the spectral line-width and spectral position of the emission lines as a polynomial function of wavelength. This wavelength correcting fitting procedure accounts for differences between the values of $T, N$, and $E$ presented from those given in Trafton et al. (1999).

The following parameters and their associated errors are returned by the fitting routine.

1. The pixel-by-pixel, wavelength-dependent, wavelength shift from the nominal wavelength returned by spectrome- 


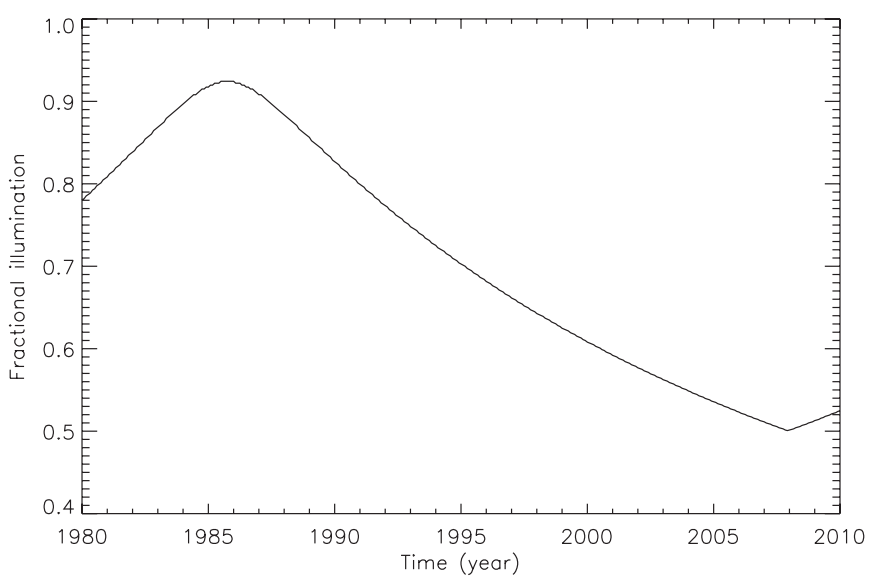

Figure 5. Time-fractional illumination of the Earth-visible hemisphere of Uranus between 1980 and 2010. The fractional illumination at solstice in 1985 does not reach unity because the maximum sub-solar latitude is $\delta_{\odot}=-82.5$.

ter software, expressed as a polynomial function of wavelength, $\Delta \lambda(\lambda)$.

2. The $\mathrm{H}_{3}^{+}$spectral line-width expressed as a polynomial function of wavelength, $\sigma(\lambda)$.

3. The $q$-LTE $\mathrm{H}_{3}^{+}$temperature, $T$.

4. The $\mathrm{H}_{3}^{+}$column density, $N$.

5. A constant background emission level, $k$.

Runtime options include the number of polynomial orders that the wavelength dependent parameters are fitted to and the ability to keep some (any) parameters at a fixed value. The $\mathrm{H}_{3}^{+}$spectral transition data come from Neale et al. (1996), which improve both the quality and quantity of the data of Kao et al. (1991). Since the total emission, $E\left(\mathrm{H}_{3}^{+}\right)$, curve of Neale \& Tennyson (1995) is only valid for temperatures greater than $1000 \mathrm{~K}$, the formulation of Miller et al. (2010) is used.

Previous workers have noted some anti-correlation between $\mathrm{H}_{3}^{+}$temperature and density (Lam et al. 1997), the extent of which is a function of the spectral $\mathrm{S} / \mathrm{N}$. Here, the linear fit to density as a function of temperature $(N=m T+b)$, given in Table 2, produces $1 \sigma$ uncertainties of the coefficients that are $50 \%$ and $75 \%$ of their actual values, respectively $(\Delta m / m$ and $\Delta b / b)$, such that there is essentially no discernible correlation. We conclude that the variations we observe are real and not a product of the fitting routine.

\section{RESULTS}

In the following sections, we investigate the long-term behavior of the column-averaged temperature $(T)$, column density $(N)$, and total energy emitted by $\mathrm{H}_{3}^{+}$over all wavelengths $(E)$, set out in Table 2. Two key properties that may have a bearing on the long-term behavior of the $\mathrm{H}_{3}^{+}$emission we see are the fraction of time that the Uranian hemisphere under observation might be illuminated by sunlight and the EUV flux that Uranus receives from the Sun. The fractional illumination of the observable disk is shown in Figure 5.

The $10.7 \mathrm{~cm}$ radiowave output of the Sun has traditionally been used as a proxy for its EUV activity. So, Figure 6 shows the nominal $10.7 \mathrm{~cm}$ flux observed at the Earth. In order to estimate what the solar EUV flux is at Uranus, the $10.7 \mathrm{~cm}$ curve is smoothed and scaled to the maximum and minimum $\mathrm{H}$ Ly $\alpha$ flux of Tobiska et al. (1997), scaled to the distance of 19 AU. In order to estimate the input into the upper atmosphere in the hemisphere visible from Earth, Figure 6 also shows the

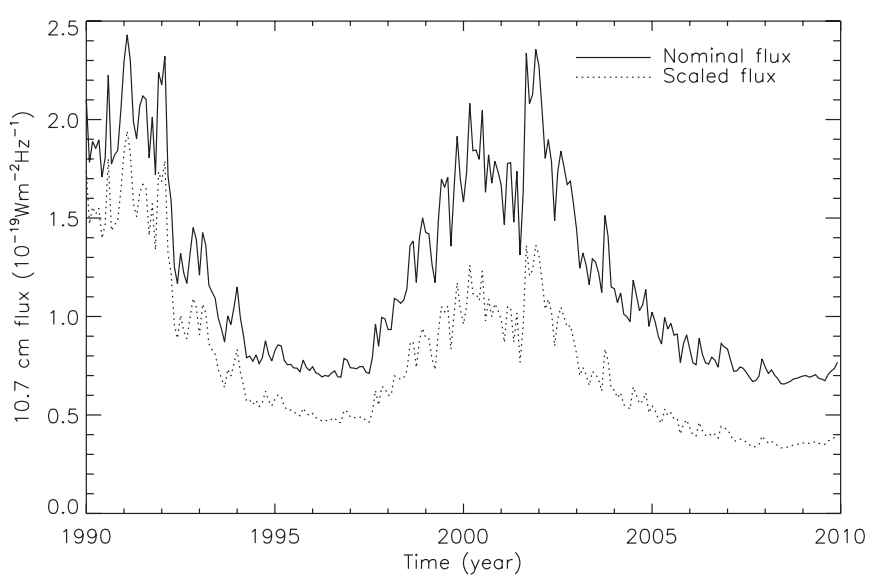

Figure 6. Solar $10.7 \mathrm{~cm}$ flux at Earth and scaled by the fractional illumination of Figure 5. Both fluxes are smoothed as to highlight the long-term variability.

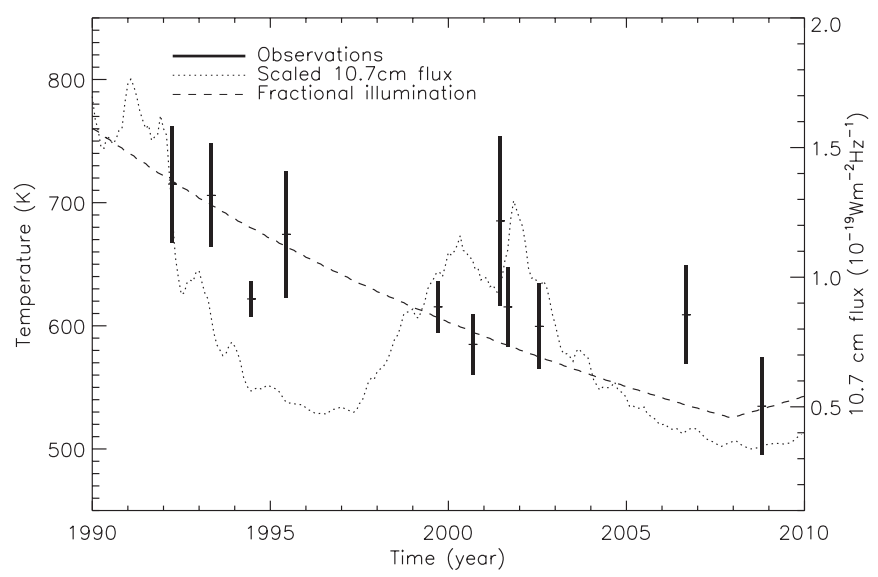

Figure 7. Long-term variability of $\mathrm{H}_{3}^{+}$temperature (solid), with the 8 month rolling average of the $10.7 \mathrm{~cm}$ flux at the Earth (dotted line). The fractional illumination (dashed line) does not have its own scale, but varies from 0.8 , in 1990 , to 0.5 , in 2008, as shown in Figure 5.

nominal $10.7 \mathrm{~cm}$ flux observed at the Earth scaled with the fractional illumination of Uranus as seen from Earth (Figure 5).

\subsection{Temperature}

Table 2 gives the temperature, $T$, averaged over each of our runs, the fitting error on that temperature, $\Delta T_{\mathrm{err}}$, and the variance in temperature, $\Delta T_{\mathrm{var}}$, on a night-by-night basis, where there are more than one night of observations in a run. Figure 7 shows the long-term variability of the $\mathrm{H}_{3}^{+}$run-averaged temperature as a function of time, together with the fractional illumination (dashed; Figure 5) and the 8 month rolling average of the scaled $10.7 \mathrm{~cm}$ flux observed at the Earth (dotted; Figure 6). The error bar on each point is either the fitting error or the night-to-night variance, whichever is the larger. The temperature of the $\mathrm{H}_{3}^{+}$ ionosphere of Uranus ranges between $715 \mathrm{~K}$ in 1992 and $534 \mathrm{~K}$ in 2008 - a reduction of $\sim 33 \%$. Although there does not appear to be a strong correlation between the fitted temperatures and the scaled $10.7 \mathrm{~cm}$ flux, there is a general fall in temperature that follows the downward course of the fractional illumination curve. Thus, the temperatures indicate a global cooling of the ionosphere as the planet rotates toward equinox.

To a first approximation, we can fit the $\mathrm{H}_{3}^{+}$temperature as a linear function of time since the 1985 solstice, $Y$ (in years), until equinox. (It is too early to see if there will be a trend for the 


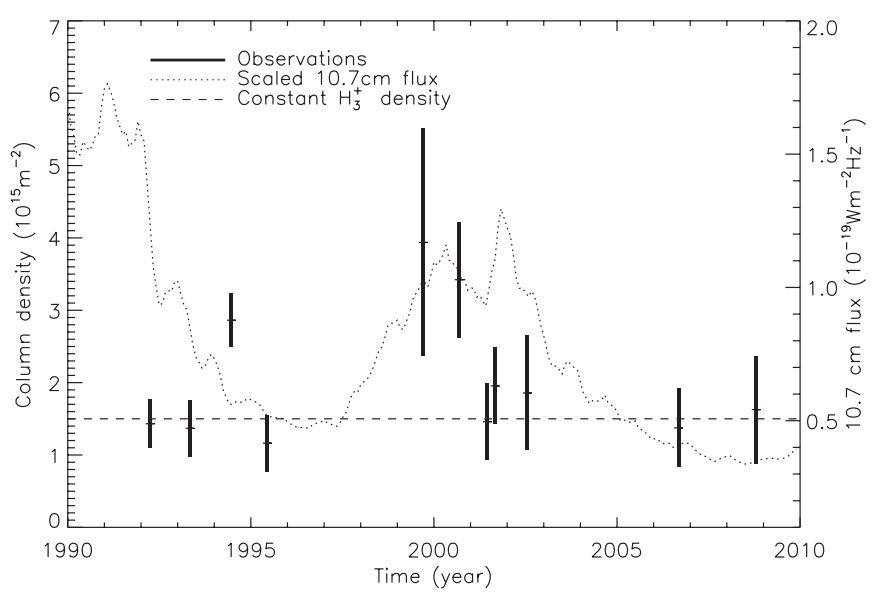

Figure 8. Long-term variability of $\mathrm{H}_{3}^{+}$column density. The dashed line shows a constant density of $1.5 \times 10^{15} \mathrm{~m}^{-2}$.

temperature to increase post equinox.) We find:

$$
T(1985.25+Y)=751.1-8.3 \times Y
$$

between 1992 and 2008. This relationship predicts the temperature at the Voyager 2 encounter of 1986 January to be $745 \mathrm{~K}$, agreeing well with the $750 \mathrm{~K}$ that Broadfoot et al. (1989) derived from UVS occultations. Equation (1) predicts the temperature of the hemisphere that is in permanent shadow at solstice to be $403 \mathrm{~K}$, but since observing the nightside is always going to be an impossible feat from the Earth, we must await the arrival of future spacecraft missions to Uranus to test this prediction.

\subsection{Column Density}

Table 2 also gives the $\mathrm{H}_{3}^{+}$column density, $N$, averaged over each of our runs, the fitting error on that density, $\Delta N_{\mathrm{err}}$, and its night-to-night variance, $\Delta N_{\text {var }}$. Note that the $\sim \pm 10 \%$ error in the absolute intensities means that $N$ may be subject to a further $\pm 10 \%$ error, over and above that given in Table 2 . This additional error also affects the total $\mathrm{H}_{3}^{+}$emission, discussed below. Figure 8 shows the column integrated $\mathrm{H}_{3}^{+}$density as a function of time, together with the $10.7 \mathrm{~cm}$ flux (dotted) and a constant column density value (dashed). What is most notable is that $N$ is remarkably constant at around $1.5 \times 10^{15} \mathrm{~m}^{-2}$ for most of the 18 year period of our observations, with just three data sets showing enhanced densities. Two of these higher density observations occur toward solar maximum, but one is close to solar minimum. And the densities observed in 1992 and 1993, when the (scaled) EUV flux should have been at its highest, fit perfectly onto the $1.5 \times 10^{15} \mathrm{~m}^{-2}$ baseline. So, there is no clear trend with either solar cycle or season or fractional illumination.

\subsection{Total $\mathrm{H}_{3}^{+}$Emission}

Finally, Table 2 also gives the total $\mathrm{H}_{3}^{+}$emission, $E$, its fitting error, $\Delta E_{\mathrm{err}}$, and its night-to-night variance, $\Delta E_{\mathrm{var}}$. The wavelength integrated emission energy of $\mathrm{H}_{3}^{+}$, or total emission, $E$, is an important parameter since it gives a good representation of the cooling-to-space due to the $\mathrm{H}_{3}^{+}$ion, the main coolant in the upper atmospheres of the giant planets. $E$ is a function both of the temperature and of the density, since the per-molecule cooling of $\mathrm{H}_{3}^{+}$increases with temperature. Figure 9 shows $E$ plotted as a function of time. The dashed line shows the calculated total emission using the temperature of Equation (1) and the constant column density of Figure 8 . It is clear that the values

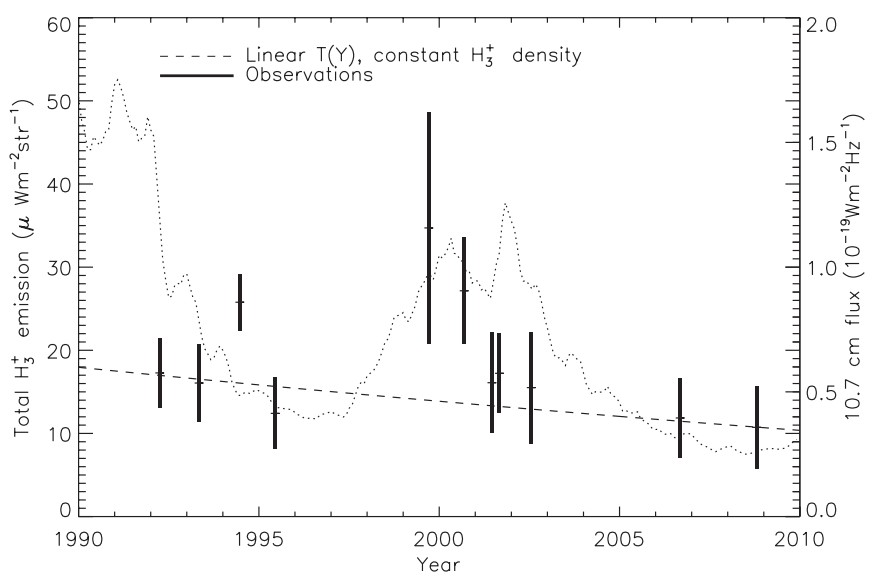

Figure 9. Long-term variability of the total energy emitted over all wavelengths, $\mathrm{E}\left(\mathrm{H}_{3}^{+}\right)$. The dashed line is the total emission of the dashed line in Figure 8 and the temperature evolution described by Equation (1).

of $E$ fit well with the temperature trend line, with the exception of data sets 3,5 , and 6 , for which high column densities have already been noted. The decrease in temperature between 1992 and 2008 leads to a fall in the total emission of $40 \%$, from $17.7 \mu \mathrm{W} \mathrm{m}^{-2} \mathrm{sr}^{-1}$ to $10.7 \mu \mathrm{W} \mathrm{m}{ }^{-2} \mathrm{sr}^{-1}$.

\section{DISCUSSION: $\mathrm{H}_{3}^{+}$PRODUCTION AND EMISSION VARIABILITY}

The overall physical conditions prevailing in the atmosphere of Uranus are a product of the solar radiation absorbed and any internal or local sources of heating and cooling (e.g., Yelle \& Miller 2004). For the upper atmosphere, the key solar wavelengths are in the ultraviolet, because these are the wavelengths absorbed in a hydrogen-rich atmosphere. Internal sources of heating may include gravity waves rising from the atmosphere below and particle precipitation from the magnetosphere above. Gravity waves, in turn, will depend on solar radiation absorbed by the middle atmosphere mainly at visible and infrared wavelengths. These heating terms will be balanced by downward conduction and cooling by radiation to space (e.g., Young et al. 2001).

The $\mathrm{H}_{3}^{+}$molecular ion is produced in a hydrogen-rich atmosphere as a result of the ionization of $\mathrm{H}_{2}$ either by photons or precipitating particles:

$$
\begin{gathered}
\mathrm{H}_{2}+h v / e^{-} \rightarrow \mathrm{H}_{2}^{+}+e^{-} / 2 e^{-} \\
\mathrm{H}_{2}^{+}+\mathrm{H}_{2} \rightarrow \mathrm{H}_{3}^{+}+\mathrm{H} .
\end{gathered}
$$

$\mathrm{H}_{2}^{+}$may also be formed as a result of charge exchange between $\mathrm{H}_{2}$ and $\mathrm{H}^{+}$so long as the difference in ionization energy can be made up: usually this is effected by having the $\mathrm{H}_{2}$ molecule vibrationally excited to $v=4$ or above.

\subsection{Solar EUV Radiation}

Since solar EUV radiation is capable of ionizing $\mathrm{H}_{2}$, thus producing $\mathrm{H}_{3}^{+}$, variations in the solar output can create variations in the observed $\mathrm{H}_{3}^{+}$emission. Consequently, variations in the solar EUV input with solar cycle phase may be an important factor in determining the intensity of the $\mathrm{H}_{3}^{+}$emission.

The total solar energy available to the upper atmosphere of Uranus, assuming an EUV albedo of 0.5 (Cochran et al. 1990), for each of the sets of observations in Table 1, can be seen in 
Table 3

The Energy Injected into the Upper Atmosphere by Solar Ultraviolet Radiation Compared to the Radiative Cooling Provided by $\mathrm{H}_{3}^{+}$

\begin{tabular}{lcccc}
\hline \hline Run Number & Year & $\begin{array}{c}\text { Solar Input } \\
(\mathrm{GW})\end{array}$ & $\begin{array}{c}\mathrm{H}_{3}^{+} \text {Cooling } \\
(\mathrm{GW})\end{array}$ & Ratio \\
\hline 1 & 1992.3 & 25.9 & 222.8 & 0.12 \\
2 & 1993.3 & 19.8 & 207.1 & 0.10 \\
3 & 1994.5 & 16.0 & 332.4 & 0.05 \\
4 & 1995.5 & 15.2 & 160.0 & 0.10 \\
5 & 1999.7 & 26.1 & 447.7 & 0.06 \\
6 & 2000.7 & 27.5 & 350.2 & 0.08 \\
7 & 2001.5 & 27.6 & 207.6 & 0.13 \\
8 & 2001.7 & 29.4 & 222.2 & 0.13 \\
9 & 2002.6 & 26.9 & 200.0 & 0.13 \\
10 & 2006.7 & 15.6 & 152.9 & 0.10 \\
11 & 2008.8 & 14.2 & 138.4 & 0.10 \\
\hline Average & $\ldots$ & 22.2 & 240.1 & 0.10 \\
\hline
\end{tabular}

Table 3. It is clear that solar input is insufficient to maintain the thermal balance of the upper atmosphere of Uranus, with $\mathrm{H}_{3}^{+}$radiating about 10 times more energy than is injected by solar EUV radiation. Therefore, there must exist additional mechanisms which deposit the bulk of the required energies into the upper atmosphere.

\subsection{Changing Illumination of the Disk}

Due to the unusual geometry of Uranus, almost all of the IAU southern hemisphere was in constant illumination during solstice (1985). At equinox (2007), the fractional illumination fell to 0.5. As noted, the temperature in Figure 7 follows the fractional illumination curve reasonably well, suggesting that season is a key factor in establishing the thermospheric temperature. Since the total cooling due to $\mathrm{H}_{3}^{+}$far exceeds the solar EUV radiation absorbed by Uranus, changes in the amount of this part of the Sun's spectrum cannot be the (sole) cause of temperature changes. It is possible, however, that seasonal effects play a part in the total amount of sunlight that is absorbed by Uranus, across the entire solar spectrum, in the atmosphere below the homopause. This may then lead to a lowering of the homopause temperature between solstice and equinox, such that cooling by downward convection is more effective at equinox. This would lead to increased heat losses from the thermosphere to the stratosphere, and a lower observed $\mathrm{H}_{3}^{+}$temperature. Young et al. (2001) have also drawn attention to this effect.

\subsection{Probability of Observing Auroral Variability}

Herbert (2009) mapped the auroral emission in the UV during the Voyager 2 encounter in 1986, which remains the only spatially resolved observation of the aurora of Uranus. The intensity and the morphology of the auroral $\mathrm{H}_{3}^{+}$emission is still unknown, and one can only guess if it maps closely to that seen in the UV in 1986. Given this lack of knowledge, the simplest assumption that can be made is that such auroral $\mathrm{H}_{3}^{+}$emission as there may be emanates from close to the magnetic poles, as is the case for both Jupiter (Satoh \& Connerney 1999) and Saturn (Stallard et al. 1999).

The fraction of a rotation period that the north or south magnetic poles are in view for a particular epoch is seen in Figure 10. It shows the northern magnetic pole rotating into view as equinox approaches, and that the northern pole becomes fractionally more visible than the southern beyond the second half of 2007. Under the assumption that $\mathrm{H}_{3}^{+}$auroral emission

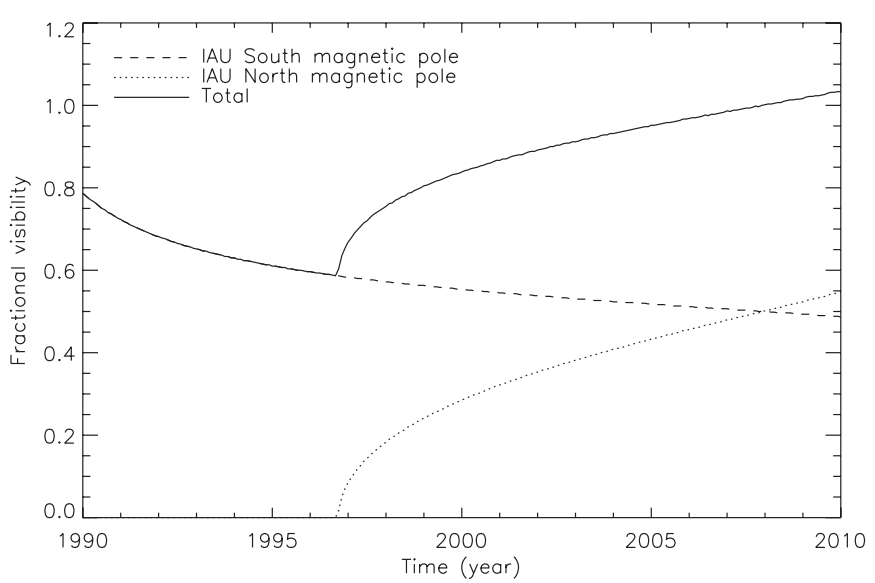

Figure 10. Fraction of a rotation period during which the north and south magnetic poles (Connerney et al. 1987) are visible from the Earth as a function of time. If both poles are visible for the entirety of a rotation the fractional visibility is equal to two (twice $100 \%$ ).

is located about the magnetic poles, the larger the fraction of a period with poles visible from the Earth, the more likely that auroral emission is observed. Figure 10 shows a minimum in the total fractional visibility of the magnetic poles in 1996 and it increasing through beyond equinox. Thus, if the auroral $\mathrm{H}_{3}^{+}$ emission on Uranus were relatively constant over time, and our observations were sampling the full range of Uranian longitudes, there would be an apparent minimum in the $\mathrm{H}_{3}^{+}$emission in 1996 as observed from the Earth. Such a trend is not seen in the data.

Instead, there are three data sets for which the column density departs significantly from the $1.5 \times 10^{15} \mathrm{~m}^{-2}$ baseline value-1994 (run 3), 1999 (run 5), and 2000 (run 6). These reach levels of $2.9 \times 10^{15} \mathrm{~m}^{-2}, 3.9 \times 10^{15} \mathrm{~m}^{-2}$, and $3.4 \times 10^{15} \mathrm{~m}^{-2}$, respectively.

Since the higher densities observed for data sets 3,5 , and 6 are not correlated with solar cycle phase, the source of ionization cannot be solar in origin and is more likely attributed to variability in particle precipitation. For Jupiter, Millward et al. (2002) found that the column density of $\mathrm{H}_{3}^{+}$varied as number of precipitating electrons, such that

$$
\log _{10} \mathrm{~N}\left(\mathrm{H}_{3}^{+}\right)=a \log _{10} F_{e}+b,
$$

where $a=0.435$ and $b=12.28$, and $F_{e}$ is the flux of incoming (10 keV) electrons measured in erg $\mathrm{cm}^{-2} \mathrm{~s}^{-1}$ and the resulting values of $N$ are also in $\mathrm{cm}^{-2}$. Unfortunately, there are no comparable model results for Uranus.

Transferring the power law found for Jupiter to the ionization of the Uranian atmosphere, however, the column density variations observed here indicate that particle precipitation would have been higher by approximately a factor of nine for the 1999 observation than the median level; in 1994, it would have been 4.6 times higher and in 2000, 6.6 times higher. This may be a sign that, for these data sets, significant auroral emission was detected. Note that the increased heating due to particle precipitation appears to be offset by increased $\mathrm{H}_{3}^{+}$and $\mathrm{H}_{2}$ cooling and downward conduction, since there is no corresponding increase in temperature.

\section{CONCLUSIONS}

In this paper, we have analyzed the behavior of the $\mathrm{H}_{3}^{+}$ ionosphere on timescales exceeding the length of a solar cycle. Our results show a general seasonal cooling of the upper 
atmosphere. The column density appears remarkably constant, although there are three particular occasions when significant enhancements are noted. There is no apparent correlation of these enhancements to the parameters investigated here. It is also clear that the $\mathrm{H}_{3}^{+}$cooling to space far exceeds the heating that can be produced by the absorption of solar EUV radiation. This is important baseline information, but it still leaves a lot to be explained.

The linear fit for the ionospheric temperature as a function of time derived here predicts a temperature of $745 \mathrm{~K}$ at the time of the Voyager 2 encounter in 1986, agreeing well with the temperature derived by Broadfoot et al. (1986). Using stellar occultations Baron et al. (1989) observed an increase in temperatures at the $1 \mu$ bar level of $8 \mathrm{~K} \mathrm{year}^{-1}$ between 1977 $(\sim 100 \mathrm{~K})$ and $1983(\sim 180 \mathrm{~K})$, as Uranus rotated toward the 1985 solstice. Subsequent to the Voyager 2 flyby, which did not yield a $1 \mu$ bar temperature (Lindal et al. 1987), Young et al. (2001) derived a temperature from a stellar occultation of $\sim 120 \mathrm{~K}$ in 1998 . The $1 \mu$ bar temperature does appear to ramp up toward solstice and is once again reduced some 13 years after equinox. It is notable that the observations analyzed here yield an identical rate of change in temperature of $8 \mathrm{~K} \mathrm{year}^{-1}$ as the $1 \mu$ bar observations prior to solstice of Baron et al. (1989).

Young et al. (2001) noted that the $1 \mu$ bar atmosphere of Uranus is not capable of radiating enough energy to produce the drop in temperature that they observed. At higher altitudes, $0.1 \mu$ bar and above, $\mathrm{H}_{3}^{+}$provides a means of efficiently cooling the planet. Our results show that this cooling ranges between $138 \mathrm{GW}$ and $448 \mathrm{GW}$ globally. Table 3 shows that the cooling is on average 10 times greater than the energy provided by solar EUV showing that the thermospheric temperature cannot be directly related to solar radiation. This energy "crisis" has been observed on all the giant planets (e.g., Melin et al. 2006, Yelle $\&$ Miller 2004), but is particularly intriguing at Uranus because of the very small internal energy source (Pearl et al. 1990).

Another species that can significantly radiatively cool the atmosphere is molecular hydrogen. Trafton et al. (1999) derived $184 \mathrm{GW}$ of global $\mathrm{H}_{2}$ emissions from Uranus in 1995 (run 4 in Table 1), observing the $\mathrm{H}_{2}$ quadrupole emissions at nearinfrared wavelengths. While this is comparable to the energy output of $\mathrm{H}_{3}^{+}$, some $\sim 70 \%$ of the $\mathrm{H}_{2}$ emissions comes from the $J=0$ and $J=1$ lines that are produced in a region just above the tropopause, such that the bulk of this energy does not contribute to the cooling of the thermosphere. Nevertheless, radiative cooling by $\mathrm{H}_{2}$ may still play an important part in determining the energy balance of the upper atmosphere of Uranus.

Ionospheric Pedersen currents are able to generate significant amounts of energy in the form of Joule heating and ion drag (Smith et al. 2005). At solstice, the northern hemisphere of Uranus is continually illuminated such that the $\mathrm{H}_{3}^{+}$component of the ionosphere is never given the opportunity to fully recombine by being rotated into the nightside. In principle, this could produce large Pedersen conductivities, enabling the transfer of more magnetospheric momentum into the thermosphere of Uranus. However, the $\mathrm{H}_{3}^{+}$densities are not largest at solstice (Figure 8).

This would suggest that if increased conductivity is driving the heating, then the heating must occur at low altitudes in the sharp hydrocarbon ion layers observed by Lindal et al. (1987) or that there must be significant quantities of $\mathrm{H}^{+}$ions that contribute to the conductivity in the higher thermosphere. Subsequent upward convection could distribute energy from electric currents flowing in the hydrocarbon ion layer into the thermosphere; downward conduction could bring heat from the $\mathrm{H}^{+}$layers of the ionosphere to those populated mainly by $\mathrm{H}_{3}^{+}$. Either one of the two additional ion sources discussed above would require "tuning" of the precipitating electron energies so that their (presumably) increased flux did not lead to a greater $\mathrm{H}_{3}^{+}$density, since this is not observed. This is more likely if a higher flux of lower energy electrons were involved, since these would cause ionization of neutral $\mathrm{H}$ atoms, without having the energy to get through to the lower levels to ionize $\mathrm{H}_{2}$, producing $\mathrm{H}_{3}^{+}$.

It is also possible, however, that the magnetospheric arrangement at solstice generates higher electric fields in the ionosphere. These would then power larger currents in the $\mathrm{H}_{3}^{+}$layers, causing more Joule heating, without the necessity of having a more conducting upper atmosphere and "specially tuned" electron energies.

The factor of two variations in $\operatorname{Ly} \alpha$ intensity observed by Clarke (1982) indicated variable aurorae are to be observed on Uranus, albeit they are fairly weak. Within each of the data sets analyzed here, we find that the variation in the total $\mathrm{H}_{3}^{+}$emission is between $13 \%$ (1994) and 46\% (2008), with an average around $33 \%$. The magnitude of these variations does not appear to be a function of solar cycle nor the season on Uranus. So, although the magnetospheric configuration changes as the planet rotates about the Sun, there probably remains a variable infrared auroral component to the uranian $\mathrm{H}_{3}^{+}$emissions whatever the season or point in the solar cycle.

This work was supported at the University of Leicester by STFC grant PP/E/000983/1 and ST/G0022223/1 for H.M. and T.S., and a RCUK Fellowship for T.S. L.M.T. acknowledges support from NASA grant NXX08A043G. T.R.G. is supported by the Gemini Observatory, which is operated by the Association of Universities for Research in Astronomy, Inc., on behalf of the international Gemini partnership of Argentina, Australia, Brazil, Canada, Chile, the United Kingdom, and the United States of America. H.M., T.S., and S.M. are visiting Astronomer at the Infrared Telescope Facility, which is operated by the University of Hawaii under Cooperative Agreement no. NNX08AE38A with the National Aeronautics and Space Administration (NASA), Science Mission Directorate, Planetary Astronomy Program. S.M. was also a Visiting Fellow at the University of Hawaii, Institute for Astronomy, Hilo, Hawaii. Part of the data presented herein were obtained at the W. M. Keck Observatory, which is operated as a scientific partnership among the California Institute of Technology (Caltech), the University of California (UC), and NASA. The United Kingdom Infrared Telescope is operated by the Joint Astronomy Centre on behalf of the Science and Technology Facilities Council (STFC) of the U.K.

Facilities: IRTF, UKIRT, Keck:II

Observing programs used in this study: UKIRT: 1992A/ 10/57, 1993A/63, 1994A/104, 1995A/4/67, 1999B/88:87, 2001A/ServH4 and 2004B/169, IRTF: 2000B/116, 2001B/ 026 and 2002A/039, 2008B/014, Keck II: 2006B/A261N2

\section{REFERENCES}

Ballester, G. E., Ben-Jaffel, L., Clarke, J. T., Gladstone, R., Miller, S., Trafton, L. M., \& Trauger, J. T. 1998, BAAS, 30, 1098

Baron, R. L., French, R. G., \& Elliot, J. L. 1989, Icarus, 78, 119

Broadfoot, A. L., et al. 1981, J. Geophys. Res., 86, 8259 
Broadfoot, A. L., et al. 1986, Science, 233, 74

Broadfoot, A. L., et al. 1989, Science, 246, 1459

Capone, L. A., Whitten, R. C., Prasad, S. S., \& Dubach, J. 1977, AJ, 215, 977

Chandler, M. O., \& Waite, J. H. 1986, Geophys. Res. Lett., 13, 6

Clarke, J. T. 1982, ApJ, 263, L105

Cochran, W. D., Wagener, R., Caldwell, J., \& Fricke, K. H. 1990, Icarus, 83, 93

Connerney, J. E. P., Acuna, M. H., \& Ness, N. F. 1987, J. Geophys. Res., 92, 15329

Desch, M. D., Connerney, J. E. P., \& Kaiser, M. L. 1986, Nature, 322, 42

Drossart, P., et al. 1989, Nature, 340, 539

Encrenaz, T., Drossart, P., Orton, G., Feuchtgruber, H., Lellouch, E., \& Atreya, S. K. 2003, Planet. Space Sci., 51, 1013

Encrenaz, T., Schulz, B., Drossart, P., Lellouch, E., Feuchtgruber, H., \& Atreya, S. K. 2000, A\&A, 358, L83

Fjeldbo, G., Kliore, A., Seidel, B., Sweetnam, D., \& Cain, D. 1975, A\&A, 39, 91

Herbert, F. 2009, J. Geophys. Res. (Space Phys.), 114, 11206

Kao, L., Oka, T., Miller, S., \& Tennyson, J. 1991, ApJS, 77, 317

Kliore, A. J., Nagy, A. F., Marouf, E. A., Anabtawi, A., Barbinis, E., Fleischman, D. U., \& Kahan, D. S. 2009, J. Geophys. Res. (Space Phys.), 114, 4315

Lam, H. A., Miller, S., Joseph, R. D., Geballe, T. R., Trafton, L. M., Tennyson, J., \& Ballester, G. E. 1997, ApJ, 474, L73

Lindal, G. F., Lyons, J. R., Sweetnam, D. N., Eshleman, V. R., \& Hinson, D. P 1987, J. Geophys. Res., 92, 14987

Majeed, T., Waite, J. H., Bougher, S. W., Yelle, R. V., Gladstone, G. R., McConnell, J. C., \& Bhardwaj, A. 2004, Adv. Space Res., 33, 197

McLean, I. S., et al. 1998, Proc. SPIE, 3354, 566

Melin, H. 2006, PhD thesis, Univ. College London

Melin, H., Miller, S., Stallard, T., \& Grodent, D. 2005, Icarus, 178, 97

Melin, H., Miller, S., Stallard, T., Smith, C., \& Grodent, D. 2006, Icarus, 181, 256
Miller, S., Joseph, R. D., \& Tennyson, J. 1990, ApJ, 360, L55

Miller, S., Stallard, T., Melin, H., \& Tennyson, J. 2010, Faraday Discuss., 147, 283

Miller, S., et al. 2000, Phil. Trans. R. Soc. A, 358, 2485

Miller, S., et al. 2006, Phil. Trans. R. Soc. A, 364, 3121

Millward, G., Miller, S., Stallard, T., Aylward, A. D., \& Achilleos, N. 2002, Icarus, 160,95

Mountain, C. M., Robertson, D. J., Lee, T. J., \& Wade, R. 1990, Proc. SPIE 1235,25

Nagy, A. F., et al. 2006, J. Geophys. Res. (Space Phys.), 111, 6310

Neale, L., Miller, S., \& Tennyson, J. 1996, ApJ, 464, 516

Neale, L., \& Tennyson, J. 1995, ApJ, 454, L169

Pearl, J. C., Conrath, B. J., Hanel, R. A., \& Pirraglia, J. A. 1990, Icarus, 84, 12

Rayner, J. T., Toomey, D. W., Onaka, P. M., Denault, A. J., Stahlberger, W. E., Vacca, W. D., Cushing, M. C., \& Wang, S. 2003, PASP, 115, 362

Sandel, B. R., et al. 1982, Science, 215, 548

Satoh, T., \& Connerney, J. E. P. 1999, Icarus, 141, 236

Smith, C. G. A., Miller, S., \& Aylward, A. D. 2005, Ann. Geophys., 23, 1943

Stallard, T., Miller, S., Ballester, G. E., Rego, D., Joseph, R. D., \& Trafton, L. M. 1999, ApJ, 521, L149

Tobiska, W. K., Pryor, W. R., \& Ajello, J. M. 1997, Geophys. Res. Lett., 24, 1123

Trafton, L. M., Geballe, T. R., Miller, S., Tennyson, J., \& Ballester, G. E. 1993, ApJ, 405, 761

Trafton, L. M., Miller, S., Geballe, T. R., Tennyson, J., \& Ballester, G. E. 1999, ApJ, 524, 1059

Yelle, R. V., \& Miller, S. 2004, in Jupiter's Thermosphere and Ionosphere, ed. F Bagenal, T. E. Dowling, \& W. B. McKinnon (Cambridge: Cambridge Univ. Press), 185

Young, L. A., Bosh, A. S., Buie, M., Elliot, J. L., \& Wasserman, L. H. 2001, Icarus, 153, 236 